\title{
Standard operating procedure for the collection and preparation of voucher plant specimens for use in the nutraceutical industry
}

\author{
Jana Hildreth • Eva Hrabeta-Robinson • \\ Wendy Applequist • Joseph Betz $\cdot$ James Miller
}

Received: 4 April 2007 /Revised: 22 May 2007 / Accepted: 25 May 2007 / Published online: 16 June 2007

(C) Springer-Verlag 2007

\begin{abstract}
A vital part of the development of any standardized protocol for the extraction of plant-derived crude extracts to be used in herbal medicine or nutritional supplementation is proper documentation of the original botanical source of the extract via acquisition of a voucher specimen. The purpose of this document is to serve as an accepted protocol for voucher specimen collection, handling, and storage, with specific guidelines to address commercial and research uses.
\end{abstract}

\author{
J. Hildreth $(\bowtie)$ \\ Blaze Science Industries LLC, \\ 4547 West 171 st St., \\ Lawndale, CA 90260, USA \\ e-mail: janah@socal.rr.com \\ E. Hrabeta-Robinson \\ Department of Molecular, Cell, and Developmental Biology, \\ University of California at Santa Cruz, \\ 439 Sinsheimer Laboratories, \\ Santa Cruz, CA 95064, USA \\ e-mail: Eva@TheRobinsons.com

\section{W. Applequist} \\ Missouri Botanical Garden, \\ P. O. Box 299, \\ St. Louis, MO 63166-0299, USA \\ e-mail: wendy.applequist@mobot.org \\ J. Betz \\ Office of Dietary Supplements, \\ US National Institutes of Health, \\ 6100 Executive Blvd., Room 3B01, \\ Bethesda, MD 20892, USA \\ e-mail: BetzJ@OD.NIH.GOV

\section{J. Miller} \\ The New York Botanical Garden, \\ 200th Street and Kazimiroff Blvd., \\ Bronx, NY 10458-5126, USA \\ e-mail: jmiller@nybg.org
}

Keywords Dietary supplements · Nutraceuticals · Quality control · Vouchers

\section{Introduction}

Although the practice of collecting voucher specimens in an herbarium as a botanical reference is centuries old, the implementation of this type of documentation in the herbal products industry has only recently come under close scrutiny [1]. Botanical reference materials are preserved specimens of confirmed identity that can be used for a variety of scientific and practical purposes, such as comparison with new specimens of undetermined identity. A voucher specimen is a botanical reference material that is associated with a specific lot or batch of biomass, and, as such, serves to document the authenticity of the bulk material, either for commercial or for research purposes. The voucher must be representative of the biomass used, i.e., it must have been collected from the same population or area under cultivation as the biomass. Moreover, it must be collected at the same time: if a voucher is collected from, putatively, the same population as a lot of biomass, but in a different season or year, the chances of accidentally collecting a voucher from a similar species are drastically increased. Each unique acquisition of a lot of biomass therefore requires that a separate, representative voucher specimen be preserved for that lot. If plants harvested or collected from multiple locations are to be used for purposes such as analytical method validation, then multiple voucher specimens representing each of the collection sites will be needed.

In ordinary botanical practice, a voucher specimen includes all available aboveground portions of a representative specimen of a particular plant population, properly dried and affixed to a herbarium sheet. It is vital that 
characteristic parts of the plant (i.e., parts that are necessary for its botanical identification, often including flowers or occasionally fruits) be a part of the herbarium specimen. If desired, other retained materials from the same lot of material (e.g., whole or powdered root or other biomass), in appropriate containers, may accompany the herbarium sheet and be considered part of the voucher specimen. Such samples can provide information on the material's microscopic, organoleptic, and chemical characteristics and thereby contribute to the accuracy of characterization of bulk materials to be used in the herb and natural product industry.

Although most corporations and researchers are now aware that bulk materials should be vouchered, no generally accepted protocol exists for the proper documentation of a plant material used as a source in the creation of an extract or dietary supplement, or in research to explore chemistry and bioactivity or to validate methods. As a result, not all parties are aware of how vouchers should be obtained, preserved, and curated, and some of those who intend to voucher their materials are unwittingly using inappropriate practices that limit the utility of their vouchers [2]. Procedures proposed here for the collection of voucher specimens for commercial or research purposes such as analytical method validation are based on those used in plant taxonomy by universities, botanical research institutions, and herbaria, with the omission of details pertaining to the discovery and identification of new taxa. The protocol is designed to have broad applicability and maximum efficiency, using commonly available products and conditions.

\section{Collection, pressing, and drying}

Data pertaining to each plant specimen should be collected in a water-resistant field notebook using indelible ink or pencil. Each collection of a single species from a single locality must be given a unique identifying number at the time of collection; if multiple sheets are pressed as vouchers of that collection, all receive the same number. In ordinary botanical practice, an individual collector continues to use a single consecutive number series throughout his or her lifetime. For each collection, data to be recorded include the date, names of collectors present, location (including geographical coordinates and altitude if equipment permits), ecological notes such as habitat type and population size in natural populations, and all information about the plants themselves that may not be evident from the dried specimen (e.g., flower or bark color, distinctive odor, or the habit and size of a large plant). For cultivated material, any known cultivar name or designation should be recorded. If photographs are taken of the plant in the field, this should be noted. Location should be recorded as precisely as possible, including country, state or province, county or municipality, and a description of proximity to easily located features such as roads, junctions, and distance markers from cities/towns. Information not known at the time of collection may be filled in later. Although the format for data collection is left to personal preference, a consistent format should be followed to make sure that all necessary information to complete the voucher specimen label has been recorded [3-5].

For small herbaceous plants, the entire aboveground portion of the plant, including stems, leaves, flowers, or fruits, and at least part of the root with soil removed, should be collected intact. If the plant is too tall to fit on a standard herbarium sheet (normally given as $16.5 \mathrm{in} . \times 11.5 \mathrm{in}$. or approximately $42 \mathrm{~cm} \times 29 \mathrm{~cm}$ ) but is not very branched, it may be possible to collect a whole plant by folding it into a $\mathrm{V}$ or $\mathrm{N}$ shape [4-7]. For plants that are too large to fit onto a sheet, representative parts should be cut (e.g., a large leaf, a portion of stem with small leaves, and an inflorescence). If possible, bark should be collected from woody plants. If male and female flowers are borne separately, portions representing both sexes should be collected; if multiple developmental stages are present in a population, all should be represented (e.g., by adding a fruiting branch to a flowering individual). Specific guidance on how to prepare specimens from difficult plants is available in published guides to field procedures [5-7].

Supplies needed include a plant press and straps, a small stack of sheets of corrugated cardboard cut to the same size as the press, sheets of blotter material or felt if possible, and single sheets of newsprint paper. A plant press can be purchased or can be constructed by assembling two lattices of narrow wooden lath about 6-mm thick, with four parallel 46-cm (18-in.) strips overlaid by five parallel 31-cm (12-in.) strips and solidly attached at the intersections. Each specimen should be pressed inside an individual folded sheet of newspaper, which should be immediately marked with the collection number in indelible ink to avoid confusion of specimens. Each specimen in newspaper should be placed between two blotters (or, if necessary, extra layers of newspaper) and then between pieces of cardboard to create a "sandwich." The press should be closed as tightly as possible with the straps to prevent wrinkling and shrinkage.

Specimens should be placed in the press immediately after collection, with structures spread out for easy identification of diagnostic features. Woody specimens may relax after a few hours in the press, making it easier to rearrange them into a desirable conformation; fragile specimens need to be permanently arranged at first pressing. Some of the leaves and flowers, or a portion of a single large leaf, should be turned over to display both 
sides of the structure. Multiple layers of overlapping leaves or branches obscure detail and hinder drying; excess parts should be trimmed off as needed. Thick dry structures such as cones can be air-dried without pressing; thick fleshy parts such as roots should be sliced before pressing; and succulent parts such as cactus stems should be sliced and scraped to remove wet internal tissues. If necessary, fleshy structures that are essentially destroyed by pressing (e.g., the flowers of some orchids) can be preserved in small screw-top jars of $70 \%$ ethanol.

Drying should proceed as quickly as possible following pressing, preferably in an electric drier at temperatures between 35 and $45{ }^{\circ} \mathrm{C}$. Effective plant driers can be constructed using light bulbs; a recent design utilizes electrical space heaters $[7,8]$. The press should be placed on its side on the dryer with the folded edge of the newspapers down and the open long edge up, so that air can flow through the corrugated cardboards. As the specimens dry, the press should be tightened frequently and the blotters or similar materials between specimens should be changed regularly, every day for fleshy plants. (Blotters should not be stacked and stored while damp, or they may mold.) Drying should continue until even the thickest parts are not at all soft. If a dedicated plant dryer is not available, the press may be placed over or in front of improvised sources of warmth or airflow (e.g., heat lamps, fans). An assembled plant press and any protruding dried plant materials are highly flammable, so appropriate caution must be used. Warmth, good ventilation, and low humidity are conducive to drying; blotters should be changed twice a day if appropriate drying facilities are not available. A press should never be put in an ordinary oven, which is both too hot and too humid [7]. Dried specimens should be kept in folds of newspaper, under appropriate environmental conditions, until labels have been prepared and the specimen can be mounted.

\section{Mounting and labeling}

All voucher specimens must be assigned a unique identification number to permanently identify that collection. As noted already, the standard format for material in botanical collections consists of the collector's last name combined with his or her collection number, as recorded in the field. For specialized purposes, such as for a series of commercial collections, identification numbers in other formats may be assigned to collections as desired. These numbers are to be noted in the field collection notebook and on any accompanying paperwork and must appear on herbarium specimen labels as well as on any containers used for storage of biomass associated with the voucher specimen or extracts therefrom. Data and information from any addi- tional testing done on biomass taken from a particular collection should be labeled with the identification number so that results will always be linked to the associated voucher specimen.

The dry specimen should be attached to acid-free mounting paper using archival adhesive, supporting heavier plant portions if necessary with linen thread or strips of linen tape or polyester film. Dots of glue or strips of tape should be used only as needed, preferably on stem portions in direct contact with the paper. It is not necessary to coat the whole specimen in glue; avoid gluing or taping flowers and leaves directly. The specimen should be positioned in such a way as to leave room on one of the lower corners of the sheet for a specimen label. Separated fragments that are sturdy enough to be stored loosely can be placed in a small envelope folded from acid-free paper and affixed to the sheet; very large separate parts such as pine cones may need to be stored separately in a labeled box.

Voucher specimen labels should adhere to a standardized format to ensure that all necessary data from the field notebook are accurately and efficiently transcribed to the label. All labels, inks, and adhesives used in the labeling process should be archival to guard against degradation with time. Information on the label should include the following: name of collector, accession number (i.e., identification number), scientific name, date of collection, location of collection, all recorded habitat and morphological data, the species' common name if desired, and the name of the person identifying the plant specimen. The physical location where the voucher specimen is stored should be included as part of the label. Examples of labels can be found in many places [3-5]. Accepted practice for research specimens noted in publications is to give the voucher number (usually the collector's last name and collection number) and the herbarium code, if the specimen is placed in a public herbarium collection [9], or the name and address of the specimen storage location.

It is also useful to add label notes or a supplementary label to specimens that have been cited in a scientific publication; this not only confirms their identity, but also helps to ensure that they will be permanently preserved. If the identity of the specimen is reevaluated (or the preferred name for a species changes) after the specimen has been mounted, a "determination label" can be added to the sheet; this small slip of paper provides the name of the botanist making this change, the date, and the correct name in the opinion of that botanist.

\section{Packaging, storage, and maintenance of collections}

Care must be taken with the storage and handling of vouchers to ensure specimen longevity and maintain 
traceability. Freezing specimens for several days prior to storage, and rotating collections periodically through the freezer, is a common nonhazardous means of insect control. The temperature of the storage room should be approximately $16{ }^{\circ} \mathrm{C}$. The collection should be stored in a commercially available specially designed metal herbarium cabinet, taking care to protect the specimen from exposure to dust, light, insects, heat, and humidity. Specimens should not be placed loose into a cabinet; adequate protection can be derived from storing a small stack of specimens in a single appropriately sized folder. Folders should be removed from cabinets only as necessary to work with the material, and should be replaced promptly. Original field notebooks or copies must be permanently retained; voucher numbers should be marked on all accompanying documentation, such as copies of field notebook pages and photographs, and included in all relevant publications.

Specimens collected as vouchers for material used in scientific publications should, if at all possible, be preserved permanently and be curated at a public herbarium [9] or other collection accessible to bona fide researchers. Vouchers collected for a purely commercial purpose, such as documentation of the identity of materials used in a consumer product, should be kept for at least 5 years, and certainly beyond the expiration date of any products derived from related biomass; after enough time has passed such that a batch of product may be expected to have been consumed or discarded, the associated voucher specimens may be discarded if desired.

\section{Vouchering commercial materials}

Voucher material, by the broad definition, is any retained identifiable material from a population or lot of material whose identity is to be documented; it need not be a pressed herbarium specimen. For example, when a company purchases a batch of bulk material from a wholesaler, the seller often does not possess nor make available simultaneously collected herbarium specimens. In these instances, the purchaser may preserve a sample of the bulk material as a voucher, but must be aware of the limitations of that voucher. Often, unprocessed bulk material in commerce can be unambiguously identified by morphological and organoleptic characteristics; however, sometimes the available parts (e.g., leaves, bark, roots) have morphological characteristics consistent with multiple related species. If validated species-specific chemical or molecular identification methods are not available to demonstrate the botanical identity of these samples, they are not adequate vouchers. Scientific studies to validate analytical methods or explore chemical variation must, of course, never utilize samples whose vouchers cannot be unambiguously identified by an independent method.
Another point to be aware of is that wild-collected material making up a single commercial "batch" may have been collected by several collectors working in several different localities. In normal research practice, a separate herbarium specimen would be collected as a voucher for each locality; combining multiple gatherings into a single batch raises the possibility of intrabatch heterogeneity, but is an unavoidable fact of the botanicals trade for many species. Vouchers for such material should combine samples taken from throughout the purchased lot, to maximize the likelihood that any inclusion of multiple species will be detected. World Health Organization [10] guidelines call for sampling $10 \%$ of containers from a batch, always rounding up, with a minimum of five (or all containers if fewer than five are purchased); samples from each container should be taken from the top, middle, and bottom, then mixed and reduced in volume until an appropriately sized sample is obtained.

Voucher samples of bulk material should be stored in sealable packaging, with care taken to ensure that samples are adequately dried. Heavy-duty plastic baggies are adequate for many samples, but associated chemicals may interact with plant compounds; if preservation of original chemical content is desired for any reason, glass containers may be preferable, and samples should be stored at $-20{ }^{\circ} \mathrm{C}$ or lower if possible. Unlike herbarium specimens, bulk material samples can be mixed up or contaminated with relative ease; labels, to be included within the package, should be prepared before vouchers are removed and bagged. Labels should state the material's botanical identity, supplier, batch number, date of receipt, and as much information on geographic source, etc. as is available from the supplier. These materials can be preserved under environmental conditions similar to those for herbarium sheets, and likewise should be frozen before storage to kill any insect pests. If vouchers must be opened and handled for any purpose, care must be taken to avoid losing material or introducing foreign material into packages. Only one package should be opened at a time, and any material removed that could have been contaminated with foreign material should not be returned to the package.

If an ideal herbarium voucher cannot be preserved at the time of harvest, because the species is harvested in a season when the plant parts most valuable for identification (usually including flowers or, in some families, fruits) are not present, suppliers may offer to provide a fertile "voucher" harvested from the same vicinity in a different season. As mentioned above, specimens collected at different times are properly treated as being different collections, so that one cannot voucher another. This practice may be of some value when the "voucher" can be guaranteed to come from the same population or individual plant, such as when material is cultivated, but 
is seldom justifiable for wild-collected material. If a sterile voucher is really impossible to distinguish from other species native to the same area, what safeguards ensure that the originally collected plants and the voucher plant are of the same species? A preferable approach is to collect a sterile voucher specimen and compare it with fertile botanical reference materials (for which the inclusion of fertile specimens later obtained from the original collecting area would indeed be ideal); this may well give adequate confidence in the botanical identity of the material.

\section{Conclusion}

Voucher specimens are critical for the confirmation and documentation of the identity of research and commercial botanicals. Lack of adequate vouchering represents a fatal flaw in a scientific study, and serious legal exposure in a commercial context. Users of botanical biomass are therefore encouraged to plan for the preservation and storage of vouchers, even before materials have been acquired. Early consideration of these issues may affect choices made in material sourcing. For example, if researchers are aware that purchasing material from a commercial source will leave them with a voucher not identifiable to the species level by traditional or validated laboratory methods, they may choose to bear the added costs of collecting and vouchering material themselves or paying others to do so, thereby increasing the quality and utility of a planned study.
Acknowledgements Financial support for this project was provided by the Office of Dietary Supplements at the National Institutes of Health and by NIH grant U19 AT003264-01; W. Folk, PI. The authors also gratefully acknowledge K. Sharpless of the National Institute for Standards and Technology for helpful discussion and three anonymous reviewers for helpful comments.

\section{References}

1. Flaster T, Lassiter J (2004) HerbalGram 63:32-37

2. Slifman NR, Obermeyer WR, Aloi BK, Musser S, Correll WA Jr, Cichowicz SM, Betz JM, Love LA (1998) N Engl J Med 339 (12):806-811

3. Frank MS, Perkins KD (ed) (2007) Preparation of plant specimens for deposit as herbarium vouchers. http://www.flmnh.ufl.edu/ herbarium/voucher.htm. Cited 14 Mar 2007

4. Horton D (2003) Herbaria and specimens: What are they? http:// www.cgrer.uiowa.edu/herbarium/HerbariaAndSpecimens.htm. Cited 14 Mar 2007

5. Bridson D, Forman L (eds) (1998) The herbarium handbook, 3rd edn. Royal Botanic Gardens, Kew

6. Smith EE Jr (1971) Preparing herbarium specimens of vascular plants. Agriculture information bulletin no 348. US Department of Agriculture, Washington

7. Liesner R (2001) Field techniques used by Missouri Botanical Garden. http://www.mobot.org/MOBOT/molib/fieldtechbook/ welcome.shtml. Cited 14 Mar 2007

8. Blanco MA, Whitten WM, Penneys DS, Williams NH, Neubig KM, Endara L (2006) Selbyana 27:83-87

9. Holmgren PK, Holmgren NH, Barnett LC (1990) Index herbariorium part 1: the herbaria of the world, Regnum Vegetabile, vol 120 , 8th edn. New York Botanical Garden, New York

10. World Health Organization (1998) Quality control methods for medicinal plant materials. World Health Organization, Geneva 